\title{
Perspectivas de jóvenes universitarias durante la pandemia de COVID-191
}

\section{Perspectives of female undergraduate students during the COVID-19 pandemic}

\author{
María Cristina Osorio Vázquez² y Hans Th. A. Bressers ${ }^{3}$
}

Fecha de aceptación: 15 de enero de 2021

Fecha de recepción: 28 de febrero de 2021

Resumen - El presente artículo tiene como objetivo describir las percepciones de mujeres jóvenes estudiantes de licenciatura de instituciones públicas y privadas acerca de la educación a distancia durante el confinamiento por el COVID-19 en tres ciudades del estado de Yucatán. Se utilizó la metodología mixta de investigación, con base en entrevistas semiestructuradas y análisis de datos de fuentes nacionales e internacionales. Entre los hallazgos se registraron los desafíos de dos grupos de estudiantes en la modalidad de educación en línea durante los primeros meses de la pandemia, en 2020. La originalidad de este artículo radica en que contribuye a la indagación sobre el avance de la formación de las mujeres, a través de recolectar información primaria para poder emitir sugerencias que les permitan continuar en el sistema educativo nacional.

Palabras clave: Coronavirus, mujer, universidad, virtual, contingencia.
Abstract - This paper aims to describe the perceptions of undergraduate female students from public and private institutions about distance education during confinement due to COVID-19 in three cities in the state of Yucatan. The methodology used was mixed research methods, based on semistructured interviews and data analysis from national and international sources. Among the findings the challenges of both groups of students in the implementation of online education during the first months of the pandemic in 2020 were recorded. This paper's distinctiveness is that it contributes to research on the advancement of girls' education, through the collection of primary information, in order to make suggestions that allow them to continue in the national educational system.

Keywords: Coronavirus, woman, university, virtual, contingency. 


\section{Introducción}

ste artículo aborda las percepciones sobre las clases virtuales de jóvenes mujeres estudiantes de licenciatura en tres ciudades del estado de Yucatán, a partir de la irrupción del COVID-19, lo que ha afectado a $90 \%$ de la población estudiantil a nivel mundial (Save our future, 2020, p. 5).

Si bien este hecho ha repercutido en varones y mujeres, son estas últimas las que se encuentran más vulnerables a ser víctimas de matrimonio forzado, embarazo adolescente, inserción temprana al mercado laboral y violencia verbal y sexual al interrumpir sus estudios (Plan International, 2015; UNDP, 2015; Odhiambo, 2020), por tal motivo, en este artículo nos centraremos en la perspectiva de ellas.

El cierre de las escuelas en la mayor parte del mundo significó un cambio en la enseñanza, de presencial pasó a ser virtual, con poca o nula preparación docente para continuar con los programas de estudios utilizando las Tecnologías de la Información. En esta transición, los países en desarrollo y emergentes no contaban con la infraestructura necesaria para llegar a todos los alumnos, lo que ha significado el atraso y deserción de los estudiantes más vulnerables, quienes no tienen acceso a la educación a distancia (Annual Status of Education Report-Rural, 2020, p. 5), considerando que en estos países solo $20 \%$ de los hogares tienen acceso a internet y alrededor de la mitad cuenta con radio o televisión (Carvalho \& Hares, 2020; Dreesen et al., 2020; Hereward, Jenkins \& Idele, 2020).

En este contexto, el tener un equipo de cómputo, un celular, conexión a internet y electricidad ha sido crucial para continuar con el proceso educativo. Aunado a la brecha digital existente hay que mencionar que estos recursos se encuentran limitados mayormente para las niñas, debido a las normas de género prevalecientes en estos lugares (Girl Effect, 2018; Amaro, Pandolfelli, Sanchez \& Brossard, 2020).

Las mujeres son responsables de una variedad de tareas domésticas en México; la cantidad de trabajo puede ser extensa cuando en casa hay familiares que requieren ayuda por su edad o enfermedad, como niños, ancianos o personas con discapacidad (Osorio, 2017).

Esta pandemia reveló la necesidad de proveer entrenamiento a la plantilla docente en tecnología para la educación o "EdTech" (Rodríguez-Segura, 2020, p. 6; Muñoz, Petesch, Turk \& Thumala, 2013, p. 61) para cambiar a enfoques de enseñanza más inclusivos, atractivos y adaptables, y para priorizar el apoyo a aquellos que más lo necesitan (Save our future, 2020, p. 7).

Estos esfuerzos son necesarios debido a que se ha advertido que muchos estudiantes no se han sentido motivados a continuar su formación utilizando las plataformas educativas, lo que podría estar determinado por la falta de experiencia de los docentes en Tecnologías de la Información aplicadas a su labor, falta de acceso a internet e inclusive de energía eléctrica en algunas regiones, no contar con un equipo de cómputo con la capacidad suficiente para poder asistir a las clases virtuales, aislamiento motivado por la contingencia relacionada con el COVID-19 o, al contrario, hogares con un gran número de integrantes, cada uno de ellos realizando sus actividades en línea, lo que en algunas ocasiones podría sobrepasar los recursos familiares en megas disponibles por casa. Entre los indicadores que se han presentado está la dificultad por parte de los estudiantes para terminar las tareas (Anderson \& Perrin, 2018, p. 2), inasistencias, no abrir las cámaras aun a petición del docente y deserción.

Kostelny et al. (2016) han documentado, a través del análisis etnográfico, que los alumnos pierden interés en aprender mientras las escuelas están cerradas (Alban et al., 2020, p. 8); otras investigaciones sugieren que los que se atrasan en la escuela tienen más probabilidades de abandonar su formación posteriormente (Mendez \& Evans, 2020, p. 2), de ahí la importancia de involucrarlos durante el proceso educativo.

Durante esta crisis sanitaria mundial el distanciamiento social ha afectado a la juventud. Otra de las consecuencias del cambio de educación presencial a virtual es la pérdida del sentido de 
pertenencia, tomando en cuenta que para muchos estudiantes son las escuelas el lugar en donde pueden encontrar el apoyo y afecto cuando están fuera de sus hogares (Morales, 2015, p. 188). Asistir a sus clases virtuales pudiera ser un mecanismo de apoyo durante esta pandemia, un espacio para tener interacciones positivas con gente de su misma edad que enfrentan problemas similares a ellos, considerando que la educación está fuertemente relacionada a conductas saludables, cuidados preventivos y reducción de conductas de riesgo (Feinstein, Sabates, Anderson, Sorhaindo \& Hammond , 2006), aspectos sumamente necesarios en momentos de confinamiento y distanciamiento social.

Debido a lo anterior, basándose en la experiencia de jóvenes mujeres cursando el nivel licenciatura en el estado de Yucatán, el presente artículo pretende describir la opinión de ellas acerca del cambio de la educación presencial a en línea, de marzo a diciembre de 2020; es decir, los primeros meses de la contingencia por COVID-19, para conocer sus percepciones y de esta manera sugerir estrategias que eviten una baja en el desempeño académico y, en el último de los casos, deserción escolar.

Es significativa la investigación sobre el avance educativo de las mujeres, ya que a mayores grados académicos mayor es el progreso en sus derechos y su movilidad intergeneracional, según cifras de la Organización de las Naciones Unidas para la Educación, la Ciencia y la Cultura (UNESCO, 2020), considerando que las niñas que permanecen en la escuela aprenden a niveles significativamente más altos (Save our future, 2020, p. 13).

De esta forma, la pregunta de investigación es la siguiente: ¿Cuál es la percepción de las mujeres estudiantes de licenciatura sobre las clases virtuales implementadas en condiciones de confinamiento en el estado de Yucatán? Con este punto de partida, en la primera sección del presente artículo se define el diseño metodológico bajo el que se llevó a cabo la investigación, posteriormente se desarrolla el marco teórico utilizado, posteriormente se presentan los resultados del estudio, el análisis de la información y finalmente las conclusiones.

\section{Diseño metodológico}

Se trata de un estudio exploratorio, ya que permite examinar sucesos que no se habían presentado antes. Si bien las pandemias habían ocurrido anteriormente en la historia de la humanidad, con efectos negativos en el desempeño escolar de las mujeres, como es el caso de Sierra Leona ante la crisis del ébola, documentada por Bandiera et al., en 2019, el uso de plataformas educativas y sus efectos en el proceso educativo en las jóvenes durante confinamiento es relativamente nuevo, por lo que es necesario registrar sus experiencias para determinar la motivación de las jóvenes estudiantes de licenciatura a continuar sus estudios, tomando en cuenta el contexto de aislamiento social obligado por la pandemia de COVID-19 en el mundo y las diversas limitaciones que trajo consigo el cambio de modalidad educativa en un corto periodo.

Los análisis exploratorios nos sirven para aumentar el grado de familiaridad con fenómenos relativamente desconocidos, a fin de obtener información sobre la posibilidad de llevar a cabo una revisión más completa sobre un contexto particular, examinar problemas del comportamiento humano, identificar conceptos o variables promisorias, establecer prioridades para investigaciones posteriores o sugerir afirmaciones (postulados) verificables (Dankhe, 1986). Esta clase de acercamientos son comunes en la investigación del comportamiento, sobre todo en situaciones en las que hay poca información (Hernández, Fernández \& Baptista, 2010).

Para realizar esta indagación se trabajó con base en información primaria obtenida de tres grupos de 10 jóvenes mujeres (lo que dio un total de 30 participantes), inscritas a la universidad tanto en instituciones públicas como privadas, para comparar sus respuestas y así entender los desafíos a los que 
se enfrentan. La metodología aplicada fue mixta, conformada por el registro de su experiencia a partir de una entrevista semiestructurada, revisión de la literatura relacionada con el tema, aunado al análisis de datos secundarios recabados de diferentes instituciones nacionales, como la Secretaría de Educación Pública (SEP) en México, así como de las Naciones Unidas, particularmente de la UNESCO, entre otras fuentes de información.

La población considerada para este estudio fueron jóvenes mujeres estudiantes de licenciatura del estado de Yucatán, que según cifras de la SEP (2019) son un total de 35 495; tomando en cuenta que la población es el conjunto de todos los casos que concuerdan con una serie de especificaciones (Selltiz, Jahoda, Deutsch \& Cook , 1976), en este caso el género femenino, inscritas en el nivel licenciatura en un espacio geográfico determinado: Yucatán.

De dicha población se eligió la muestra, definida como un subgrupo (Hernández et al., 2010), conformada por tres grupos de 10 integrantes cada uno. De esta forma, la muestra se constituye como no probabilística de sujetos voluntarios, ya que la elección de las participantes no se hizo al azar, sino que dependió de un proceso de toma de decisiones por parte de ellas.

La implementación de la entrevista semiestructurada se realizó por medio de tres asistentes de investigación; ellos la enviaron vía WhatsApp y correo electrónico a sus redes de contacto, quienes contestaron el cuestionario, por lo tanto, la composición de la muestra estaba fuertemente interconectada con una relación de confianza entre el entrevistador y las entrevistadas.

Se consideró el perfil establecido en la muestra: mujeres jóvenes entre 18 y 23 años, que es el rango de edad de quienes asisten regularmente a la universidad, en instituciones ubicadas en el estado de Yucatán. Sin embargo, es relevante mencionar que entre la población se puedan encontrar otras mayores o menores que cursan este nivel de estudios.

Los lugares en los que se llevó a cabo este estudio fueron la ciudad de Mérida, capital de Yucatán; Maxcanú, ubicada a 68 kilómetros al oeste de la ciudad capital, e Izamal, a $67 \mathrm{~km}$ al este de la ciudad de Mérida (Figura 1).

\section{Figura 1.}

Ubicación de Mérida, Izamal y Maxcanú, en la península de Yucatán, México

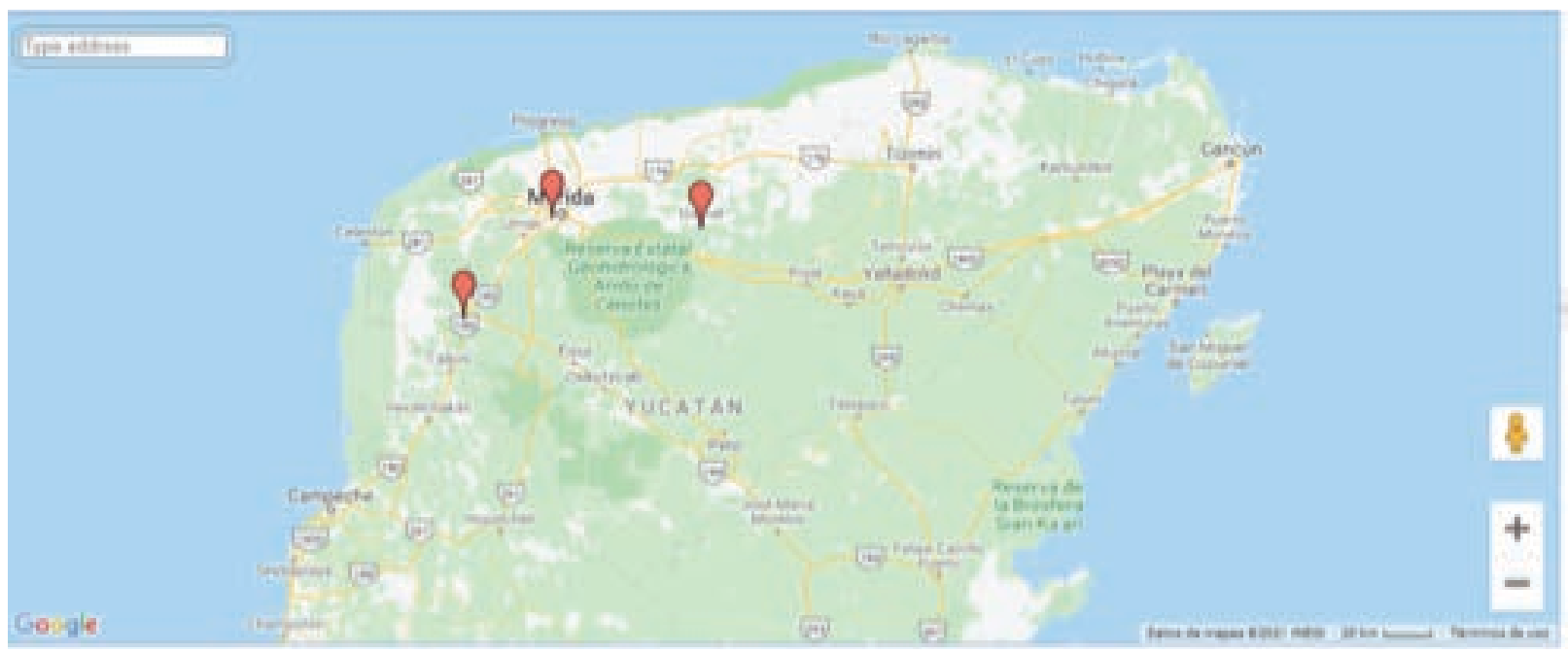

Fuente: Elaboración propia. 
Siendo personas conocidas de los entrevistadores, la participación de las jóvenes se llevó a cabo voluntaria y anónimamente, sin firmar un consentimiento informado para colaborar en el estudio. Este tipo de muestra dirigida usualmente es utilizada en las ciencias sociales y en los estudios de la conducta de los seres humanos, idónea para analizar las percepciones y motivación, como en este artículo.

El hecho de que la entrevista fuera anónima permitió una mayor libertad en las respuestas referentes a la adaptación al aula virtual, con sus aciertos y contratiempos. Se determinó que la muestra no probabilística era la adecuada para efectuar este estudio exploratorio, ya que posibilitó registrar las experiencias de las participantes en tiempos de restricción en la movilidad debido a la pandemia.

\section{Marco teórico}

Considerando que este artículo trata sobre la motivación de las estudiantes de licenciatura en tres diferentes ciudades del estado de Yucatán, el marco teórico se fundamentó en teorías relacionadas con el comportamiento humano, es decir, qué es lo que activa, dirige y mantiene la conducta de los seres humanos en una amplia variedad de situaciones (Agut \& Carrero, 2007), sobre todo en condiciones que podrían minimizar su rendimiento, por ejemplo, el desempeño escolar de las participantes durante la pandemia.

En primera instancia se abordará la teoría del condicionamiento operante de Skinner (1953), quien afirmó que toda conducta humana está determinada por sus consecuencias; en este caso se relacionará con la asistencia y permanencia en el aula virtual para aprobar la materia (reforzamiento positivo) o bien la inasistencia, el involucramiento de los familiares, reprobación de la materia, deserción escolar como reforzamiento negativo, lo cual podría aplicarse a las experiencias de los estudiantes independientemente de que asistan a universidades públicas o privadas.

A la luz de la teoría de Skinner, las personas realizan acciones no porque el proceso sea una parte de su autoexpresión, sino para evitar los castigos identificados como reforzamientos negativos.

Por otra parte está la teoría de las necesidades de McClelland (1961), que establece que hay dos tipos de necesidades que impulsan al ser humano para hacer ciertas tareas: las primarias y las secundarias (entre éstas, las sociales, como la afiliación). Esta teoría se utilizó para entender la importancia de la afiliación en las jóvenes participantes de este estudio; debido a su rango de edad de 18 a 23 años, dichas necesidades determinan en gran medida la motivación y las acciones, lo cual se encuentra presente en diferentes niveles socioeconómicos.

Los amigos representan uno de los grupos primarios más importantes para los jóvenes en esta edad, en algunos casos prevalece sobre la familia, que es identificada como el conjunto primario de mayor importancia. En los grupos primarios hay una alta intensidad afectiva, alto grado de cohesión, cooperación íntima e identificación con el grupo; ejercen una poderosa influencia en la construcción del "yo social", esto es, la imagen como el individuo cree ser percibido por los demás (Ballenato, 2014).

También se utilizaron la teoría de la expectativavalencia de Vroom (1964) y la teoría del establecimiento de metas de Locke y Latham (1990), ambas relacionadas con fijar y cumplir objetivos, lo que quiere decir que la motivación está intrínsecamente vinculada con el logro de las metas, el tiempo y los recursos que se van a invertir en ellas.

Alcanzar los objetivos propuestos implica, de manera natural, un proceso paulatino de resolución de problemas; adicionalmente requiere de competencias sociales, como capacidad de diálogo y escucha, tolerancia y paciencia, toma de decisiones y firmeza para ejecutarlas, además de otras cualidades en el terreno personal de la autovaloración y la confianza para encarar los conflictos (Morales, 2015, p. 192). En este caso, la meta de las jóvenes participantes sería aprobar las materias y, en última instancia, concluir los estudios de licenciatura, tomando en cuenta que las 
habilidades anteriormente citadas deben adaptarse al contexto digital.

Si bien los propósitos son los mismos entre las estudiantes que asisten a universidades públicas y privadas, esta teoría subraya los recursos invertidos para lograrlos, los cuales son diferentes en este estudio exploratorio, aspecto que se desarrollará más en las siguientes secciones.

\section{Resultados}

A continuación se expondrán los resultados del estudio. Como se mencionó anteriormente, el nivel socioeconómico define en gran medida la disponibilidad de recursos, los cuales son especialmente necesarios para el proceso de aprendizaje en situaciones de confinamiento. El primer grupo lo conforman alumnas que acuden a universidades privadas; entre sus medios de comunicación durante la contingencia predominó el WhatsApp; sin embargo, también mencionaron zoom, google meet, house party, skype y facetime. Entre las principales preocupaciones ante el cierre de las escuelas de todos los niveles en México, en marzo de 2020, expresaron el no ver a sus amigos y no entender las clases. Una de ellas comentó: Considero que no todos aprenden igual y muchas materias no son para tomarlas en línea, ya que surgen dudas que se necesitan resolver con un maestro, como es el caso de Contabilidad y Matemáticas.

De la misma forma, manifestaron que la comunicación no es la misma utilizando aplicaciones, ya que se podría malinterpretar el mensaje, lo que en algunos casos ha conducido a la pérdida de amistades, como compartió otra de las participantes: He perdido amistades por malos entendidos, no es lo mismo hablar por mensaje, los tonos y la manera en que cada quien se expresa y se entienden los mensajes depende de la persona. También los problemas de comunicación han estado presentes con los docentes, como menciona otra alumna: Quisiera regresar a clases presenciales porque la comunicación con los docentes sería más adecuada.
Entre los retos que se han enfrentado al recibir clases en línea están los siguientes: exceso de tareas, aburrimiento, cansancio, fallas en el internet o en la electricidad, dificultad para entender el tema, distraerse fácilmente, pérdida de tiempo al inicio de la clase mientras todos se conectan, falta de flexibilidad y paciencia de los maestros, y el hogar como lugar inapropiado para tomar la clase. Una de ellas explica: Considero que no todos aprenden igual y muchas materias no son para tomarlas en línea, ya que surgen dudas que se necesitan resolver con un maestro. De igual forma, las señales de internet son diferentes, pudiera estar lloviendo en casa de algún alumno mientras que en la casa de los demás no y el profesor no puede detenerse por ese alumno si le falla el internet. Muchos profesores no tienen la paciencia necesaria para impartir clases en línea y deberían de ser conscientes de que las plataformas y el internet fallan, no todos los alumnos aprenden igual y no es lo mismo clases presenciales a tenerlas a través de una pantalla. También, muchos hogares no son aptos para aprender, ya que se vive con muchas personas, los demás igual pueden estar tomando clases en línea y el internet se puede alentar aún más o también haber problemas en casa que hagan que los alumnos se desconcentren o se cohíban con sus padres (presión de que sean buenos alumnos).

Lo que más extrañan es convivir con su grupo de amigos durante clase y en los recesos, y la interacción con el personal de la escuela, como indica otra participante: El encierro muchas veces no te ayuda a tranquilizarte, a veces necesitas apoyo de algún maestro, ya sea moral o profesional, y es más fácil; el contacto con gente te ayuda a despejar tu mente, pasas ratos agradables con tus amigos.

El segundo grupo lo integraron 20 estudiantes que asisten a escuelas públicas. Existen algunas similitudes en sus respuestas respecto de quienes están inscritas en universidades privadas, como extrañar a sus amigos; no obstante, en su contestación se nota mayor cohesión social, aunque sea a la distancia, como 
lo dijo una de ellas: Mis amigos en cualquier momento que los necesite están para ayudar, esto coincide con respuestas de otras participantes, como: Queremos socializar; Debido al confinamiento, buscamos la forma de platicar; Antes no con todo el grupo platicabas, ahora nos apoyamos. Su opinión se explica con base en la teoría de la motivación de McClelland (1961), quien asume que las principales causas impulsoras de los seres humanos están relacionadas con las necesidades de afiliación.

Entre las habilidades desarrolladas durante la contingencia, las integrantes del primer grupo que asisten a universidades privadas mencionaron mayormente la paciencia; en el segundo grupo las respuestas fueron más diversas, como pintar, tejer, bordar, hacer manualidades, cocinar, maquillarse, mejorar el dominio del idioma inglés, autosuficiencia y organización.

En el aspecto en el que más difirieron ambos grupos fue en las dificultades que encontraron en el proceso de cambio de enseñanza presencial a virtual. Si bien en el segundo grupo que asisten a escuelas públicas también refirieron su preocupación por no entender los temas, facilidad para distraerse en línea y cansancio por estar sentadas frente a un monitor-como comentó una de ellas: Estando en línea me distraigo mucho y no comprendo mis clases completamente, sus inquietudes están relacionadas con no poder continuar sus estudios, lo que no se registró en el primer grupo.

Entre las respuestas del segundo grupo se encontraron: Me preocupa no poder continuar con mis estudios; Pensé que perdería un semestre. También manifestaron falta de recursos para poder aprender en línea, como una computadora eficiente y señal de wifi. De igual manera, se habló de una sobrecarga de responsabilidades aunadas a las normas de género de su contexto: Nos dejan demasiadas tareas más las actividades del hogar, lo que no se escuchó en el primer grupo.

Asimismo, se declaró temor de regresar a las aulas al transportarse a sus centros de estudios, como en el siguiente testimonio: Creo que es muy peligroso para aquellos que viajan o que estudian de tarde, ya que aún se debe de cumplir con todas las medidas de salud, por ejemplo el cubrebocas y digamos que también los camiones no llevan a mucha gente; corres el peligro de que te pueda pasar algo porque no reconoces a la persona que va a tu lado, por estar usando el cubrebocas, así como llegar tarde a tu casa por la cuestión de los camiones, y estar muy de noche andando sola en la calle.

El transporte ha sido determinante para el avance educativo de las mujeres, más aún en las zonas rurales alejadas de los centros urbanos (Osorio, 2017), esta intranquilidad va más allá del recurso económico al que se encuentra relacionado, sino que refiere al desplazamiento de las mujeres de los centros de estudio a sus hogares de manera segura. Al respecto, es importante decir que entre las estudiantes que acuden a instituciones privadas no se mencionó el miedo a ser agredidas en el transporte público, sobre todo porque pueden utilizar automóviles particulares, ya sean propios, de familiares o de amigos.

Otra situación que se exteriorizó, pero esta vez entre las universitarias que asisten a escuelas privadas, es el mayor involucramiento de los padres, quienes muchas veces toman la clase con sus hijas, lo que contribuye a brindar una mejor atención por parte de las autoridades educativas (Annual Status of Education Report [Rural], 2020, p. 23).

\section{Análisis de los resultados comparados con las teorías de la motivación}

En las respuestas de las participantes se notó un proceso de adaptación lento hacia la educación virtual, sumado a una sobrecarga de tareas encargadas por los profesores. Una de las situaciones que ocurrió en el traslado del modelo presencial al virtual es que muchos docentes no tenían el entrenamiento ni los recursos necesarios para poder desarrollar su clase mediante una plataforma educativa, lo que se sustituyó con proyectos y tareas que cubrían los contenidos. Esto lo manifestaron ambos grupos de encuestadas. 
La asistencia a las aulas virtuales no las motiva hoy en día, se sienten cansadas e inclusive se expresó que dejan a la computadora hablando sola. Debido a lo anterior se puede afirmar que, de acuerdo con las entrevistadas, continuaron sus estudios no porque estuvieran motivadas, sino que -como establece la teoría del condicionamiento operante de Skinner (1953)- el reforzamiento positivo que obtienen al asistir a sus clases virtuales es la oportunidad de ver a sus amigos, mientras que la reprobación y represalias familiares, como regaños y castigos de sus padres, funcionan a manera de un refuerzo negativo.

De esta forma, el estudio es más un esfuerzo mecanicista alejado de la alegría que refirieron que sentían cuando asistían a la universidad e iban a la biblioteca a estudiar con sus amigos. Según lo establece la teoría de las necesidades de McClelland (1961), este sentido de pertenencia es necesario para satisfacer las necesidades de afiliación en la juventud, como sucede en las participantes en este estudio.

La teoría de la expectativa-valencia de Vroom (1964) y la del establecimiento de metas de Locke y Latham (1990) nos ayudan a entender por qué las alumnas continúan asistiendo a sus clases virtuales si les parecen tan desmotivantes, ellas esperan que la contingencia sea temporal y pronto puedan regresar al aula para terminar sus estudios. Sin embargo, si bien prefieren la educación presencial sobre la virtual, investigaciones sostienen que la implementación de modelos híbridos podría ser una alternativa cuando se retorne a los salones de clases (Alban et al., 2020, p. 20).

La comunidad educativa a nivel mundial está explorando estas opciones y pese a que la pandemia relacionada al COVID-19 ha significado un atraso educativo significativo con impacto en generaciones por venir, también es la oportunidad de replantear los modelos de educación para que sean innovadores, incluyentes y que contribuyan a adquirir habilidades para la inserción al mercado laboral.

Se ha avanzado en el desarrollo de habilidades relativas a las tecnologías de la educación, tanto en docentes como en estudiantes, a partir de la necesidad de continuar independientemente de las difíciles condiciones que imperaban al cierre de las escuelas, en marzo de 2020. Infraestructura, equipo de cómputo e inclusive un espacio en el hogar son necesarios para poder llevarlo a cabo.

\section{Conclusiones}

Este estudio se realizó para conocer cuáles son las perspectivas de las jóvenes universitarias acerca de la educación en línea implementada por la crisis sanitaria, tanto en universidades públicas como en privadas.

Ambos grupos estuvieron de acuerdo en que la educación en línea no es la apropiada para comprender las clases numéricas, pero las preocupaciones de las alumnas de las instituciones públicas están más relacionadas con la falta de recursos para continuar sus estudios, por ejemplo fallas en la red y no tener una computadora eficiente. Sin embargo, también se evidenció una mayor cohesión social entre las estudiantes de escuelas públicas para resolver las situaciones que se les han presentado durante estos meses de confinamiento.

Uno de los efectos más preocupantes de la pandemia es el número de niñas que no regresarán a las aulas, por lo que esta situación ofrece la oportunidad de reflexionar sobre cómo podemos mejorar las posibilidades para que ellas retornen. El gobierno mexicano ya está invirtiendo en infraestructura para que el internet llegue a las zonas más remotas del país, queda a la sociedad mexicana cambiar para que las normas de género no detengan su avance educativo. Que las niñas decidan su futuro en lugar que otros lo decidan por ellas (UNESCO, 2020).

\section{Referencias}

Agut, S. \& Carrero, V. (2007). Contribuciones al estudio de la motivación laboral: enfoques teóricos desde la dimensión de autoexpresión del ser humano. Revista de Psicología del Trabajo y de las Organizaciones, 23(2), 203- 
225. Recuperado de https://www.redalyc.org/ pdf/2313/231317597003.pdf

Alban, C., Akseer, S., Dreesen, T., Kamei, A., Mizunoya, S. \& Rigole, A. (2020). COVID-19: Effects of School Closures on Foundational Skills and Promising Practices for Monitoring and Mitigating Learning Loss. Florencia: UNICEF. Recuperado de https://www.unicef-irc.org/publications/1144-covid19-effects-of-school-closures-on-foundational-skills-and-promising-practices.html

Amaro, D., Pandolfelli, L., Sanchez-Tapia, I. \& Brossard, M. (2020, August 4). COVID-19 and education: The digital gender divide among adolescents in sub-Saharan Africa. UNICEF. Recuperado de https://blogs.unicef.org/evidence-for-action/ covid-19-and-education-the-digital-genderdivide-among-adolescents-in-sub-saharan-africa/

Anderson, M. \& Perrin, A. (2018). Nearly one-infive teens can't always finish their homework because of the digital divide. Pew Research Center. Recuperado de https://www.pewresearch.org/ fact-tank/2018/10/26/nearly-one-in-five-teenscant-always-finish-their-homework-because-ofthe-digital-divide/

Annual Status of Education Report (Rural). (2020). Wave 1. Recuperado de http://www.asercentre. org/Wave/p/373.html

Ballenato, G. (2014). Trabajo en equipo. Dinámica y participación en los grupos. Madrid: Ed. Pirámide.

Bandiera, 0., Buehren, N., Goldstein, M., Rasul, I., \& Smurra, A. (2019). The economic lives of young women in the time of Ebola: Lessons from an empowerment program. Recuperado de http://documents1.worldbank.org/curated/ en/452451551361923106/pdf/WPS8760.pdf

Carvalho, S. \& Hares, S. (2020, July 22). Six ways COVID-19 will shape the future of education. Cen- ter for Global Development. Recuperado de https://www.cgdev.org/blog/six-ways-covid-19-wiII-shape-future-education

Dankhe, G. (1986). Investigación y comunicación. Madrid: McGraw Hill.

Hernández, R., Fernández, C. \& Baptista, P. (2010). Metodología de la Investigación. México: Ed. McGraw Hill.

Dreesen, T., Akseer, S., Brossard, M., Dewan, P., Giraldo, J., Kamei, A., Mizunoya, S. \& Santiago, J. (2020). Promising practices for equitable remote learning: Emerging lessons from COVID-19 education responses in 127 countries. UNICEF. Recuperado de https://www.unicef-irc.org/publications/ pdf/IRB\%202020-10\%20CL.pdf

Feinstein, L., Sabates, R., Anderson, T., Sorhaindo, A. \& Hammond, C. (2006). What are the effects of education on health? Recuperado de http:// www.oecd.org/education/innovation-education/37437718.pdf

Girl Effect. (2018). Real girls, real lives, connected: A global study of girls' access and usage of mobile, told through 3000 voices. Recuperado de HYPERLINK "https://prdgirleffect-corp.s3.amazonaws.com/documents/GE_VO_Full_Report $\% 20$ compressed.pdf" https://prdgirleffect-corp. s3.amazonaws.com/documents/GE_VO_Full_Report compressed.pdf

Hereward, M., Jenkins, R. \& Idele, P. (2020). Remote learning amid a global pandemic: Insights from MICS6. UNICEF. Recuperado de https://blogs.unicef.org/evidence-for-action/remote-learning-global-pandemic-insights-mics6/

Kostelny, K., Lamin, D., Manyeh, M., Ondoro, K., Stark, L., Liley, S. \& Wessells, M. (2016). 'Worse than the War': An Ethnographic Study of the Impact of the Ebola Crisis on Life, Sex, Teenage 
Pregnancy, and a Community-Driven Intervention in Rural Sierra Leone. Save the Children. Recuperado de https://resourcecentre.savethechildren. net/library/worse-war-ethnographic-study-impact-ebola-crisis-life-sex-teenage-pregnancy-and-community

Locke, E. \& Latham, G. (1990). A theory of goal setting and task performance. EUA: Prentice Hall.

McClelland, D. (1961). The achieving society. Princeton: Van Nostrand Reinhold.

Osorio, M. (2017). Understanding Girls' Education in Indigenous Maya Communities in the Yucatán Peninsula. Implications for Policy and Practice. US: Brookings.

Mendez, A. \& Evans, D. (2020). COVID-19 and Girls' Education: What We Know So Far and What We Expect. Recuperado de https://www.cgdev. org/blog/covid-19-and-girls-education-what-weknow-so-far-and-what-we-expect-happen

Morales, M. (2015). El logro de las metas y la consolidación de los proyectos de vida de los y las jóvenes egresados/as de la Telesecundaria Tetsijtsilin en Tzinacapan, Cuetzalan, Puebla. Bajo el volcán, 15(22), 179-208. Recuperado de https:// www.redalyc.org/pdf/286/28642148012.pdf

Muñoz Boudet, A. M., Petesch, P., Turk, C. \& Thumala, A. (2013). On norms and agency: Conversations about gender equality with women and men in 20 Countries. World Bank Group. Recuperado de https://openknowledge.worldbank.org/ bitstream/handle/10986/13818/768180PUBOEPIOOICOOPUBODATE04012013.pdf?sequence=1\&isAllowed=y

Selltiz, C., Jahoda, M., Deutsch, M., \& Cook, S. (1976). Métodos de investigación en las relaciones sociales. Madrid: RIALP.

Skinner, B. (1953). Science and human behavior. New York: Free Press.

Vroom, V. H. (1964). Work and motivation. New York: Wiley \& Sons. 